\title{
Quantifying the contamination by old main-sequence stars in young moving groups: the case of the Local Association ${ }^{\star}$
}

\author{
J. López-Santiago ${ }^{1,2}$, G. Micela ${ }^{2}$, and D. Montes ${ }^{1}$
}

\author{
1 Departamento de Astrofísica y Ciencias de la Atmósfera, Universidad Complutense de Madrid, 28040 Madrid, Spain \\ e-mail: [jls; dmg]@astrax.fis.ucm.es \\ 2 INAF - Osservatorio Astronomico di Palermo Giuseppe S. Vaiana, Piazza Parlamento 1, 90134 Palermo, Italy \\ e-mail: giusi@astropa.unipa.it
}

Received 23 December 2008 / Accepted 23 February 2009

\begin{abstract}
Context. The associations and moving groups of young stars are excellent laboratories for investigating stellar formation in the solar neighborhood. Previous results have confirmed that a non-negligible fraction of old main-sequence stars is present in the lists of possible members of young stellar kinematic groups. A detailed study of the properties of these samples is needed to separate the young stars from old main-sequence stars with similar space motion, and identify the origin of these structures.

Aims. Our intention is to characterize members of the young moving groups, determine their age distribution, and quantify the contamination by old main-sequence stars, in particular, for the Local Association.

Methods. We used stars possible members of the young ( 10-650 Myr) moving groups from the literature. To determine the age of the stars, we used several suitable age indicators for young main sequence stars, i.e., X-ray fluxes from the Rosat All-sky Survey database, photometric data from the Tycho-2, Hipparcos, and 2MASS database. We also used spectroscopic data, in particular the equivalent width of the lithium line $\mathrm{Li}$ I $\lambda 6707.8 \AA$ and $\mathrm{H}_{\alpha}$, to constrain the range of ages of the stars.

Results. By combining photometric and spectroscopic data, we were able to separate the young stars (10-650 Myr) from the old ( $>1$ Gyr) field ones. We found, in particular, that the Local Association is contaminated by old field stars at the level of 30\%. This value must be considered as the contamination for our particular sample, and not of the entire Local Association. For other young moving groups, it is more difficult to estimate the fraction of old stars among possible members. However, the level of X-ray emission can, at least, help to separate two age populations: stars with $<200 \mathrm{Myr}$ and stars older than this.

Conclusions. Among the candidate members of the classical moving groups, there is a non-negligible fraction of old field stars that should be taken into account when studying the stellar birthrate in the solar neighborhood. Our results are consistent with a scenario in which the moving groups contain both groups of young stars formed in a recent star-formation episode and old field stars with similar space motion. Only by combining X-ray and optical spectroscopic data is it possible to distinguish between these two age populations.
\end{abstract}

Key words. Galaxy: stellar content - Galaxy: solar neighborhood - stars: kinematics - stars: activity - stars: coronae

\section{Introduction}

It is well known that star formation takes place inside giant molecular clouds and, indeed, stellar class I and class II objects (classical T Tauri stars, cTTS) are found mainly in these regions. Young stars are strong X-ray emitters. In particular, cTTS and weak-line T Tauri stars (wTTS) have emission levels above those observed for main-sequence (MS) stars. Thus, X-ray surveys should preferentially detect cTTS and wTTS inside the forming regions. In a study of the spatial distribution of X-ray coronal emitters detected with ROSAT, Guillout et al. (1998) found different over density regions of X-ray active stars coinciding with the position of the nearby molecular complexes: Chamaleontis I and II $(d \sim 100 \mathrm{pc})$, Taurus-Auriga $(d \sim 140 \mathrm{pc})$, ScorpiusCentaurus-Lupus $(d \sim 150 \mathrm{pc})$, and Ophiuchus $(d \sim 160 \mathrm{pc})$. In contrast, other regions exhibited the average Galactic plane characteristics. It is logical to expect post-T Tauri stars to be found in the proximity of these stellar complexes, but not many

\footnotetext{
* On-line Tables with the data are only available in electronic form at the CDS via anonymous ftp to cdsarc.u-strasbg.fr $(130.79 .128 .5)$ or via
}

http://cdsweb.u-strasbg.fr/cgi-bin/qcat?J/A+A/499/129
post-T Tauris are observed near the molecular clouds (Herbig 1978). Mamajek \& Feigelson (2001) pointed out that the main process by which stars are dispersed, i.e., the evaporation of stellar clusters and/or stellar complexes, is a slow process, but high dispersal velocities are required to transport stars some tens of parsecs from their parental clouds on short timescales. A dispersion velocity of $1-2 \mathrm{~km} \mathrm{~s}^{-1}$ is sufficient to separate a star from its formation locus by as much as $10 \mathrm{pc}$ in 5-10 Myr, and, thus, many young stars may travel to considerable distances from their parental clouds. Since many of the nearby star-forming regions are situated in the southern hemisphere, many young pre-mainsequence stars (PMS) stars with low declinations are found in the solar neighborhood (Torres et al. 2006).

A series of associations of late-type stars with ages ranging from 8 to $50 \mathrm{Myr}$ and having similar space motion have been discovered in our neighborhood: TW Hya, $\beta$ Pic, AB Dor, $\eta$ and $\epsilon$ Cha, Octans, and Argus associations, and the Great Austral complex (GAYA), which includes the TucanaHorologium, Columba, and Carina associations (Zuckerman \& Song 2004; Torres et al. 2008). A detailed study of the space motion of these young associations has shown that many of them were close to a molecular cloud in the past, in particular 
the Scorpius-Centaurus-Lupus complex (Zuckerman \& Song 2004) and the dense clouds in Ophiuchus and Corona Australis (Makarov 2007; Fernández et al. 2008). Thus, a significant portion of the young stellar population in the solar vicinity is a remnant of the star formation that took place in the past in the region of the Galaxy occupied by Sco-Cen-Lup.

Slightly older groups of stars ( 50-650 Myr) with similar space motion have also been detected in the solar neighborhood. They are the classical stellar kinematic groups, or moving groups (see Montes et al. 2001a; López-Santiago et al. 2006, and references therein). In contrast to the young stellar associations, the stars belonging to a moving group are situated all over the sky. The dispersion velocity and the differential Galactic rotation, acting together over millions of years, have caused the wide separation of their members. A large spread in the velocity space of these stars is also observed (Skuljan et al. 1999). The idea of moving groups consisting of coeval stars is rather controversial. The over density of stars in some regions of the $U V$-plane could also be the result of dynamical perturbations caused by spiral waves (e.g. Famaey et al. 2005). However, several works showed that different age subgroups are situated in the same region of the Galactic velocity plane as the classical moving groups (see Asiain et al. 1999). Famaey et al. (2007) studied a large sample of stars sharing the space motion of the Hyades cluster, and determined that part of these stars were surely associated to it in the past, while the remainder are older stars trapped at resonance. Together with the result afore mentioned this suggests that those regions of the $U V$-plane consist of both field-like stars and young coeval ones (Famaey et al. 2007, 2008; Antoja et al. 2008; Klement et al. 2008; Francis \& Anderson 2009; Zhao et al. 2009). This problem is not exclusive to moving groups, but also to stars in young T associations. Thus, Bertout \& Genova (2006) found that among the stars with similar proper motions in the Taurus region, there are field stars and pre-main-sequence ones. These authors also noticed that it is impossible to distinguish kinematically between pre-main sequence and field stars in their sample and that it is crucial to remove possible interlopers before searching for a moving group of young stars. In particular, distinguishing between members - i.e., coeval stars - of the moving groups and other field-like stars with similar space motion is necessary to investigate properly their contribution to the stellar population of the solar neighborhood.

Studies of the stellar content in flux-limited shallow X-ray surveys (Favata et al. 1993; Micela et al. 2007; López-Santiago et al. 2007) have detected an excess of yellow stars in observations that cannot be reproduced by standard galactic models using any form of continuous star-formation rate. A possible explanation in terms of binary systems with a yellow primary star and a secondary $M$ dwarf, which responsible for the X-ray emission, was proposed by Micela et al. (2007). However, this does not explain the apparent excess of stars with high X-ray fluxes detected in the $\log N-\log S$ diagram (López-Santiago et al. 2007). This group of high X-ray emitters also have low scale height, which is typical of young stars. The optical follow-up of the coronal sources in the Einstein Medium Sensitivity Survey (Sciortino et al. 1995) demonstrated that many of them are indeed young lithium-rich stars. A similar result was obtained for the North Ecliptic Pole (NEP) survey (Affer et al. 2008). It agrees with a scenario in which the solar neighborhood consists of a standard population of stars formed with a constant star-formation rate, and an additional young stellar population. In this case, we should be able to detect this young population from their X-ray emission, since young stars are high X-ray emitters. A different
Table 1. Young moving groups.

\begin{tabular}{lcc}
\hline \hline Moving group & Age $[\mathrm{Myr}]$ & Associated cluster(s) \\
\hline Local Association & $10-300$ & Pleiades, $\alpha$ Per, M34, \\
& & $\delta$ Lyr, NGC 2516, IC 2602 \\
IC 2391 Supercluster & $80-250$ & IC 2391 \\
Castor & 200 & - \\
Ursa Major & $300-500$ & Ursa Major \\
Hyades Supercluster & 650 & Hyades, Praesepe \\
\hline
\end{tabular}

question is how to explain their presence in the solar vicinity and their origin.

In this work, we investigate the contamination by old mainsequence stars in samples of possible members of the young moving groups. Our main goal is to quantify the contribution of old stars in the list of candidates. We use optical photometric and spectroscopic data, as well as X-ray data from the ROSAT satellite. We attempt to determine the nature of these stars using the information given by different age indicators such as the lithium line at $6707.8 \AA$, the X-ray emission level, and the chromospheric activity. Isochrone fitting is used as well to place constraints on the age spread in the young moving groups. In particular, we explore the Local Association age spread caused by the higher number of candidates in our sample.

\section{Data compilation}

In our study, we considered late-type stars proposed to be members of the young stellar kinematic groups of Montes et al. (2001a). This sample includes only late-type (spectral types $\mathrm{F}-\mathrm{M}$ ) field stars in the solar neighborhood - typically at distances $d \leq 100 \mathrm{pc}$ - selected by the authors from several compilations of the literature (see Montes et al. 2001a, for a complete bibliographical list and the selection criteria). Although the sample is biased towards active stars because it was compiled mainly from studies of magnetic (chromospherical and coronal) activity and surveys of young late-type stars, many stars were taken from works in which no differentiation was made between old and young stars, such as the catalog of the 100 nearby stellar systems given by the Research Consortium on Nearby Stars (RECONS ${ }^{1}$ ), or the search for kinematic groups in the solar neighborhood by Orlov et al. (1995). After the compilation, Montes et al. (2001a) used only kinematical criteria to assign each star to a moving group. A list of the young moving groups is given in Table 1, together with the stellar clusters historically associated with each group. We also give the age - or range of ages - of each moving group given in the literature, i.e., the Local Association (Asiain et al. 1999), Hyades supercluster (Skuljan et al. 1999), Ursa Major moving group (Soderblom \& Mayor 1993; Asiain et al. 1999; King et al. 2003), IC 2391 supercluster (Eggen 1991), and Castor moving group (Barrado y Navascués 1998). In general, the ages were determined by isochrone fitting of the members of the moving groups, together with some spectroscopic criteria, such as the lithium abundance and chromospheric activity. To the initial sample of 535 stars, we added the 21 members of the moving groups from the spectroscopic survey of late-type stars in the solar neighborhood of López-Santiago (2005) and López-Santiago et al. (2009) that were not included in Montes et al. (2001a). Therefore, our sample contains a total of 556 latetype stars. López-Santiago et al. (2009) determined the lithium abundance, rotational and radial velocity, and level of magnetic

\footnotetext{
${ }^{1}$ RECONS: http://www . recons .org/
} 

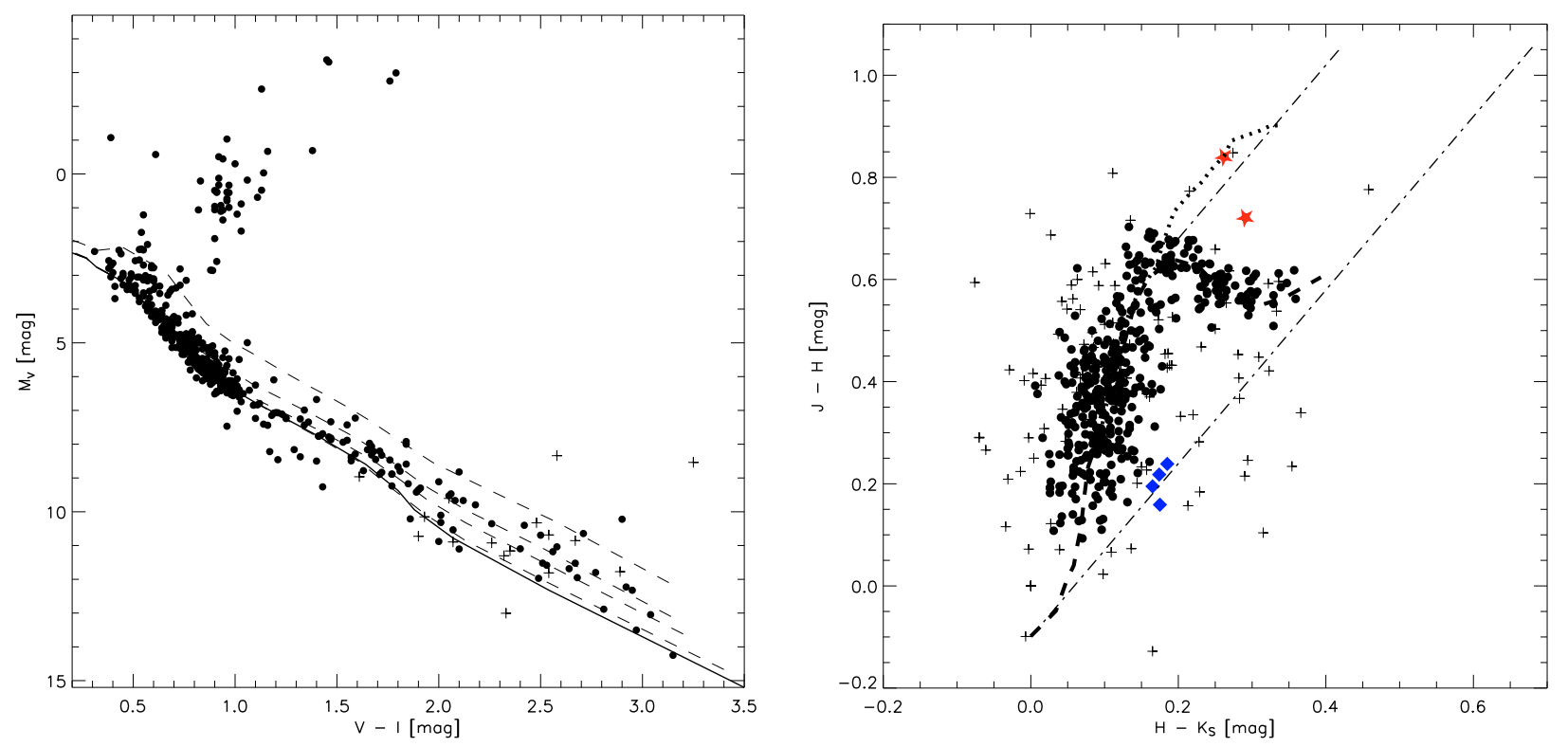

Fig. 1. Left: $M_{\mathrm{V}}$ vs. $V-I$ of the sample of stars possible members of the young moving groups in the Hipparcos catalogue. Filled circles are the stars with reliable photometry. Crosses are stars with uncertain $\sigma_{V-I} \geq 0.1 \mathrm{mag}$. Pre-main sequence isochrones of Siess et al. (2000) are plotted as dashed lines (from top to bottom: 10, 20, 50, and $120 \mathrm{Myr}$ ), while the continuous line represents the ZAMS. The dot situated over the $10 \mathrm{Myr}$ isochrone is AT Mic, which is a visual binary. Hipparcos gives the visual magnitude of the system. Assuming that both stars in the system have spectral type M 4.5, the correction of $M_{\mathrm{V}}$ would be $\sim 0.75$, situating AT Mic just on the $10 \mathrm{Myr}$ isochrone, which is coherent with its membership in the $\beta$ Pictoris moving group. Right: near-IR color-color diagram of the stars in our sample. Filled circles are stars with good quality $2 \mathrm{MASS}$ photometry (quality flag = "AAA"). The star-like symbols represent FK Ser and HD 142764. The filled grey diamonds are stars with precise photometry situated out of the main sequence track. Stars with error-bars larger than 0.1 mag are plotted as crosses. Dashed and dotted lines are the main-sequence track and the giant branch defined by Bessell \& Brett (1988), transformed here to the 2MASS system. The dotted-dashed lines represent the reddening vector for $\mathrm{A} 0$ and $\mathrm{M} 0$ dwarfs.

activity of a sample of 144 late-type stars members of the moving groups, using data from echelle spectrographs. For these 144 stars, we applied different age indicators to our data from high resolution $(\Delta \lambda / \lambda \sim 50000)$ optical observations.

Optical photometric information $\left(B_{\mathrm{T}}-V_{\mathrm{T}}\right.$ and $V-I$ colors) was taken from the Tycho-2 and Hipparcos catalogues (ESA 1997; $H g$ et al. 2000). The Tycho- 2 colors $\left(B_{\mathrm{T}}-V_{\mathrm{T}}\right)$ were used to determine $V$ magnitudes following the Sect. 1.3 of the Hipparcos Introduction and Guide to the Data (ESA 1997). We note that the values of the $V-I$ colors in the Hipparcos catalogue are given in the Cousin photometric system. Of the 556 stars in the initial sample, only 12 do not have Tycho- 2 entries, while 62 are not included in the Hipparcos catalogue. The information on the distance provided by Hipparcos was combined with the visual magnitude $(V)$ of each star to determine its absolute visual magnitude $M_{\mathrm{V}}$. For the stars not included in the Hipparcos catalogue, we used the distances given in Montes et al. (2001a), who either took them from the literature or determined spectroscopic parallaxes. We searched for IR counterparts by crosscorrelating our sample with the $2 \mathrm{MASS}^{2}$. We initially used a search radius of $r=10$ arcsec, although only 9 IR sources (2\% of the sample) were then found at $r>5$ arcsec. It is remarkable that no IR source was found for 6 stars with the chosen radius, all of which are faint dwarfs $(V>11 \mathrm{mag})$. One hundred and six IR counterparts show large errors in the 2MASS colors $(\sigma \geq 0.1 \mathrm{mag})$. This was taken into account when studying their position in the near-IR color-color diagram (see Sect. 3).

We also searched for X-ray counterparts of the stars in both the ROSAT All-Sky Survey Bright Source Catalogue (RASSBSC) and the Faint Source Catalogue (RASS-FSC). A search

2 The Two Micron All Sky Survey (2MASS) is available at http://www . ipac. caltech. edu/2mass/database. radius of 30 arcsec was adopted, bearing in mind the ROSAT X-ray object coordinate determination accuracy. A total of 341 stars (61\% of the moving-groups sample) are matched with $\mathrm{X}$-ray sources, out of which about $6 \%$ are expected to be spurious (see Guillout et al. 1998, for a detailed discussion). Since we are concerned with statistical studies, a contamination of $6 \%$ ought not to affect any of the conclusions drawn in this paper. To determine the X-ray fluxes, we used the count rateto-energy flux conversion factor (CF) relation found by Schmitt et al. (1995):

$\mathrm{CF}=(\mathrm{A} \cdot \mathrm{HR}+\mathrm{B}) \times 10^{-12} \mathrm{erg} \mathrm{cm}^{-2}$ counts $^{-1}$

where HR is the hardness-ratio of the star in the ROSAT energy band $0.1-2.4 \mathrm{keV}, A=5.30$, and $B=8.31$. We note that this $\mathrm{CF}$ relation is valid for main-sequence stars. Hünsch et al. (1996) found that for late-type giants and super-giants, $B=8.7$. X-ray fluxes were determined by multiplying the $\mathrm{CF}$ by the count-rate of the sources in the same band. Fluxes were later transformed into luminosity using the distances of the stars. Since the CF and the count rate (CR) are defined for the ROSAT energy band $0.1-2.4 \mathrm{keV}$, the X-ray luminosity $L_{\mathrm{X}}$ is also defined in this band.

\section{Evidences for the presence of an old population}

In Fig. 1 (left panel), we plot $M_{\mathrm{V}}$ versus $V-I$ for the possible members of the moving groups. Pre-main sequence (PMS) isochrones of 10, 30, 50, and $120 \mathrm{Myr}$ of Siess et al. (2000) are overplotted as dashed lines, while the continuous line represents the ZAMS. Our stars are situated mainly on the main-sequence (MS) locus, but a large quantity are located above the ZAMS. Evolved (giant) stars are distinctive because of their high luminosity. The results are compatible with the sample containing a 
mixture of MS, PMS and some evolved stars. The PMS population shows a range of ages of approximately 10-120 Myr, as deduced by comparing the data with the isochrones.

Only one T Tauri star (FK Ser) is known to be present in the sample, but it is not plotted in the $M_{\mathrm{V}}$ versus $V-I$ diagram because we did not find any value of $V-I$ in the literature. In the near-infrared color-color diagram (Fig. 1, right panel), there are only two stars with precise 2MASS photometry, i.e., the already mentioned FK Ser and HD 142764, a K5 dwarf with $A_{V}=1.8 \mathrm{mag}$ (Eiroa et al. 2001) that are situated above the MS. The remaining stars are on or close to the MS. Some stars with photometry of lower accuracy (plotted as crosses in the figure) are outside the MS track, but their position could be an artifact of the imprecise photometry. It is interesting to note that two of the four stars near the reddening vector (filled diamonds) host "hot jupiters", while the other two are known to be PMS stars.

In Fig. 2, we plot $L_{X}$ versus $V-I$ for the sources in our sample. For comparison, we also plot the stars in the Hipparcos catalogue at $d \leq 100 \mathrm{pc}$ with a ROSAT counterpart (small dots). In a similar way to Zickgraf et al. (2005), we observe a clear trend of $L_{X}$ decreasing with decreasing stellar temperature in our sample, but we also observe that the sources are divided into two strips (or branches): the lower envelope of the first one coincides with the locus of the $\log L_{\mathrm{X}} / L_{\mathrm{bol}}=-5$ relation (dashed line); in contrast, the sources in the upper strip are situated in the region delimited by the $\log L_{\mathrm{X}} / L_{\mathrm{bol}}=-3$ relation (grey area), corresponding with the saturation value for active stars. A similar behavior was noticed by Daemgen et al. (2007) using other colors in a more restricted sample of field stars (see their Fig. 2). It has been observed that the gap in the $L_{X}$ versus $V-I$ diagram is a consequence of the decrease in X-ray emission with age (see again Daemgen et al. 2007, for a complete discussion). The upper envelope of the $\log L_{\mathrm{X}} / L_{\mathrm{bol}}$ distribution is populated by stars exhibiting lithium absorption lines, typical of young stars, while the lower branch is typically populated by older stars without lithium absorption line. The combination of both X-ray emission and lithium abundances allows us to distinguish between old and young stars in the main sequence.

\section{Old star population contamination in the Local Association}

Due to the large number of possible members in our sample, the Local Association is the most suitable moving group for quantifying the fraction of old stars among the candidate members of the moving groups. It is also the moving group that contains candidates with a higher spread in ages and differences in the stellar properties. We used the information compiled by us from the literature (see Sect. 2) to determine the physical properties and, in particular, the age of each candidate.

In Fig. 3, we plot the equivalent width of the lithium line $\lambda 6707.8 \AA$ versus the $V-I$ color of the stars considered to be members of the Local Association. For comparison, we also plot the upper envelopes of some clusters with accurately determined ages. From the figure, we can identify two populations: the first one has equivalent widths similar to or even higher than those of the stars of the Pleiades, and the second one has equivalent widths that are below the Hyades upper envelope. Most of the stars in the first group exhibit also strong X-ray emission, the filled circles representing stars with $\log L_{\mathrm{X}} / L_{\text {bol }} \geq-4$. They also have high levels of chromospheric activity. Figure 4 presents the flux in the $\mathrm{H}_{\alpha}$ line in the candidates of the Local Association and its relation with the X-ray luminosity. Moreover, the stars

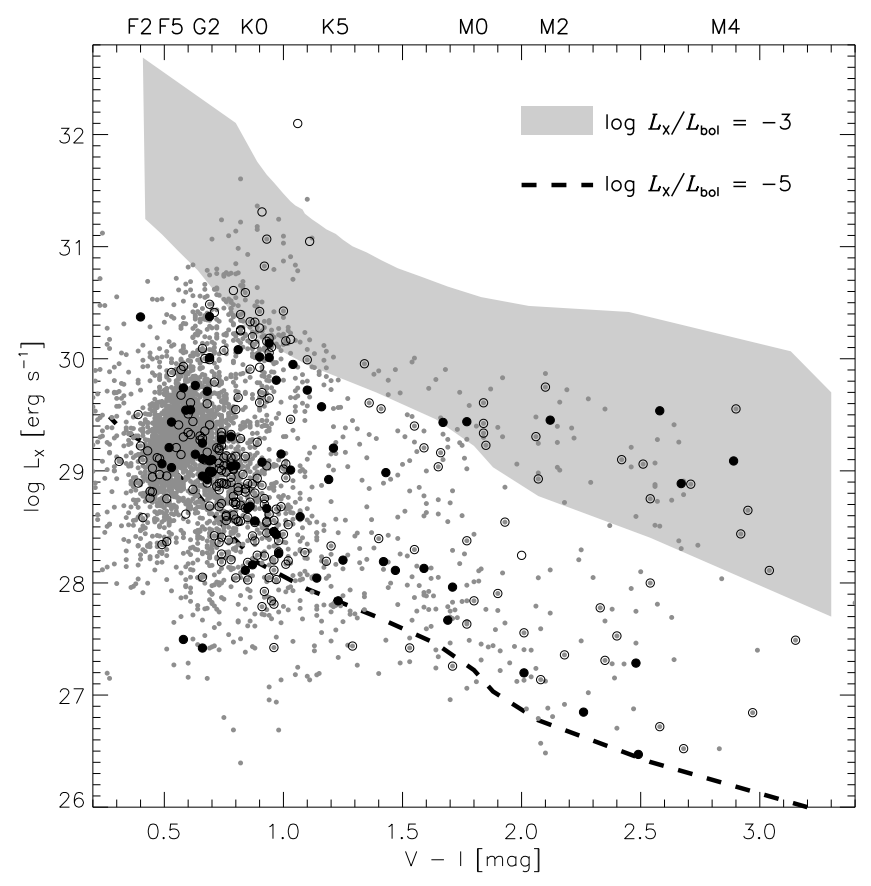

Fig. 2. X-ray luminosity vs. $V-I$ color diagram for the stars in our sample (circles). Filled circles are the stars with high-resolution spectroscopic observations. Small dots are the stars from the Hipparcos catalogue with $d \leq 100 \mathrm{pc}$. The grey area represents the region of $\log L_{\mathrm{X}} / L_{\mathrm{bol}}=-3$, with the lower edge corresponding with the ZAMS of the pre-main sequence models of Siess et al. (2000), and the upper edge with the $1 \mathrm{Myr}$ isochrone. The dashed line represents the $\log L_{\mathrm{X}} / L_{\mathrm{bol}}=-5$ relation.

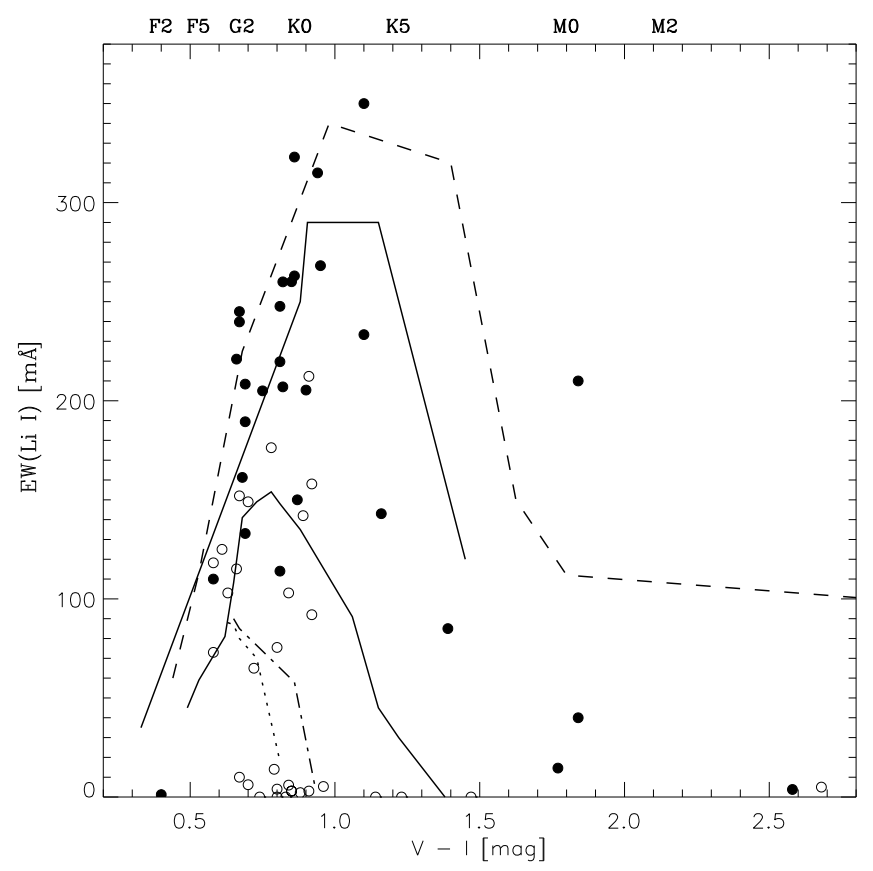

Fig. 3. Lithium ( $\lambda 6707.8 \AA$ line) equivalent width of the stars of the Local Association. The upper envelopes of the stars of some stellar clusters are plotted: dashed line for IC 2391 (10-35 Myr; Montes et al. 2001b), continuous line for the Pleiades (80-120 Myr; Soderblom et al. 1993), doted-dashed line for the Coma Berenices cluster ( 400 Myr; López-Santiago 2005), and dotted line for the Hyades ( $650 \mathrm{Myr}$; Soderblom et al. 1993). The lower envelope of the Pleiades is also plotted as a continuous line. The filled circles are stars with $\log L_{\mathrm{X}} / L_{\mathrm{bol}} \geq-4$. 

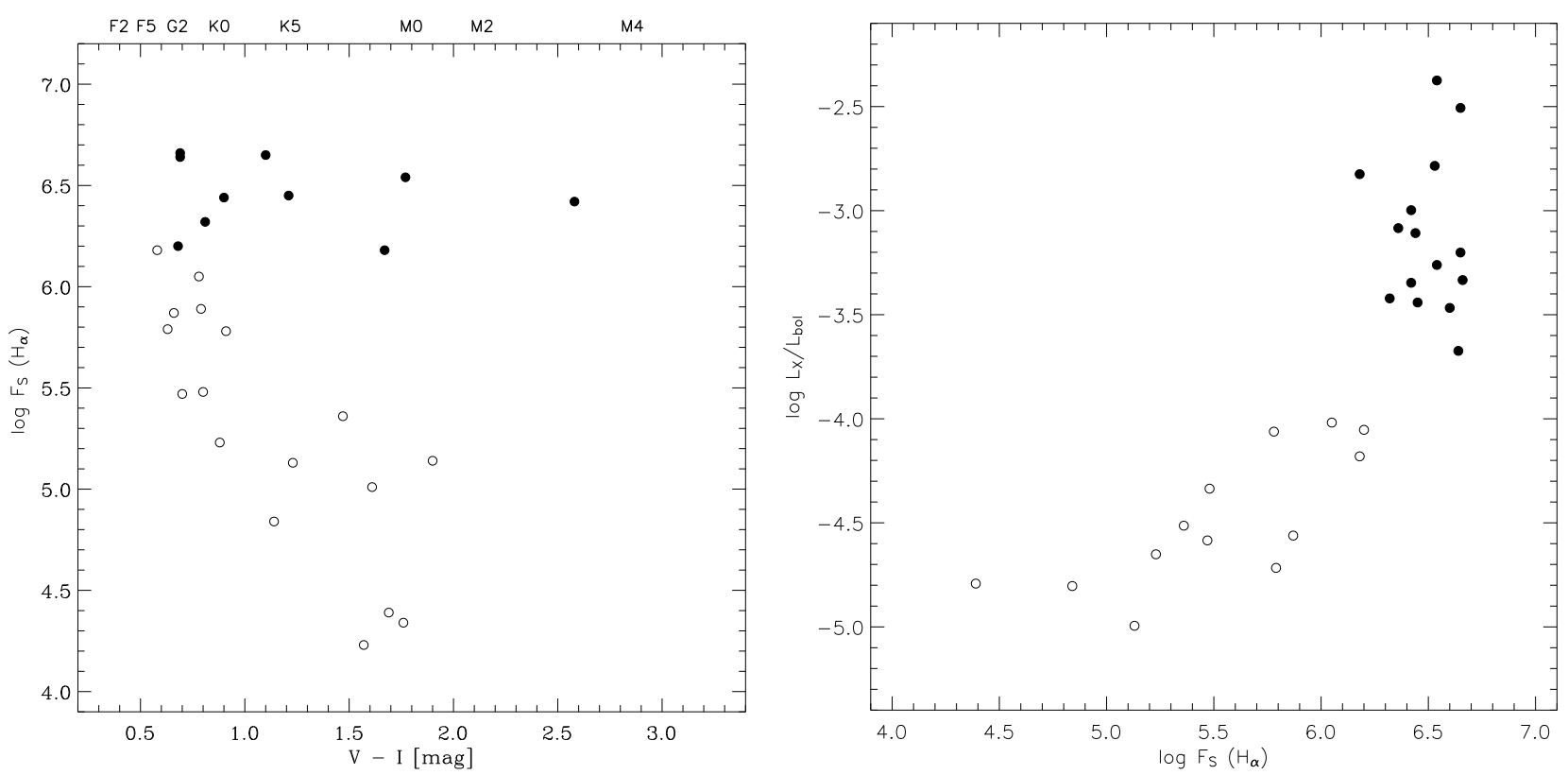

Fig. 4. Left: $\log F_{\mathrm{S}}\left(\mathrm{H}_{\alpha}\right)$ vs. $V-I$ of the members of the Local Association for which we have a measure of the equivalent width of $\mathrm{H}_{\alpha}$. The filled circles are stars with $\log L_{\mathrm{X}} / L_{\text {bol }} \geq-4$. Right: $\log L_{\mathrm{X}} / L_{\mathrm{bol}}$ vs. $\log F_{\mathrm{S}}\left(\mathrm{H}_{\alpha}\right)$ of the members of the Local Association. The symbols are the same as in the left panel.

with high X-ray emission levels exhibit a saturation in the $\mathrm{H}_{\alpha}$ flux at each spectral type (Fig. 4, left panel). All these results are compatible with the stars with higher equivalent widths of the lithium line Li I in our sample being, at least, as young as the Pleiades (indicated by the upper and lower envelopes in Fig. 3). Although it is impossible to estimate their age, its range must be 10-120 Myr. The stars with equivalent widths of Li I below the lower envelope of the Pleiades show levels of chromospheric and X-ray emission typical of field stars. This group of stars is dominated by non-members of the moving group.

Assuming that all stars with low X-ray and chromospheric emission levels (and low equivalent widths of lithium in FGK stars) are non-members of the Local Association, we find that the contamination of the initial sample of candidates is $\sim 50 \%$. This number, nevertheless, must be taken as an upper limit since our sample is incomplete. In particular, the number of matches between our sample and the RASS decreases with increasing distance. At $d \leq 40 \mathrm{pc}$, approximately $70 \%$ of our stars are cross-identified, while at $d \leq 50 \mathrm{pc}$, only a half of them have a RASS counterpart. This suggests that some X-ray emitters at large distances are undetected because of the flux limit of the RASS, producing a bias in our sample. We cannot reject these stars a priori as members of the moving groups. Using only the stars at $d \leq 40 \mathrm{pc}$, for which we are more complete, the contamination of our sample by non-members is $\sim 30 \%$, which we propose to be a more reliable value. We note that our result is valid only for the candidates identified by Montes et al. (2001a) and is closely related to the adopted selection criteria. Famaey et al. (2008) observed a higher percentage of field stars in a sample of $\mathrm{K}$ and $\mathrm{M}$ giant stars in the Pleiades Moving Group. Bertout \& Genova (2006) also presented a detailed study of the TaurusAuriga $\mathrm{T}$ association, where the authors identified 53\% of field stars among 217 possible pre-main sequence stars in this region.

\section{Contamination of the other moving groups}

We cannot apply the above analysis to the other moving groups since their number of candidates is too small to draw robust conclusions. Furthermore, the other moving groups are, on average, older than the Local Association and the age indicators that we have used are effective only for ages less than few hundreds million years. The lithium abundance, for instance, decays very rapidly for late- $\mathrm{K}$ and $\mathrm{M}$ dwarfs and may be barely detectable at the age of the Pleiades for late-type stars, while X-ray and chromospheric activity indicators may even be used at slightly older ages. In agreement with their age, only the members of the Castor Moving Group show higher values of equivalent width of Li I, but the number of stars for which we have data is not high enough to reach robust conclusions.

Studying the X-ray emission can still help us to separate stars of different age. In Fig. 5, we plot $\log L_{\mathrm{X}} / L_{\mathrm{bol}}$ versus $V-I$ for all stars of our sample with RASS counterparts. Each moving group is represented by a different symbol. The two continuous lines indicate the median X-ray emission of Pleiades and Hyades members determined by us from ROSAT data. The high X-ray emitters of the Local Association exhibit X-ray emission levels above those of the Pleiades members. A similar behavior is observed in the Castor Moving Group. Both the Local Association and the Castor candidates seem to show a similar fraction of contaminating old field stars. The $\mathrm{dK}$ and $\mathrm{dM}$ of the Ursa Major Moving Group (300-500 Myr) have $\log L_{\mathrm{X}} / L_{\text {bol }} \sim-4$, between the Castor Moving Group and the Hyades Supercluster, which have low $L_{\mathrm{X}} / L_{\mathrm{bol}}$ ratios. However, for $\mathrm{dF}$ and $\mathrm{dG}$ stars, it is more difficult to identify this effect, because of the poor sensitivity X-ray luminosity to age. Finally, the late-type stars in the IC 2391 Supercluster show high X-ray emission. This would agree with the IC 2391 being as young as the Pleiades. However, the number of candidates in this moving group is quite small and we cannot draw significant conclusions.

\section{Summary and conclusions}

We have attempted to quantify the contamination by old field stars among candidate members of the young stellar kinematic groups, or moving groups. Our sample was selected from possible members of the young stellar kinematic groups studied by 


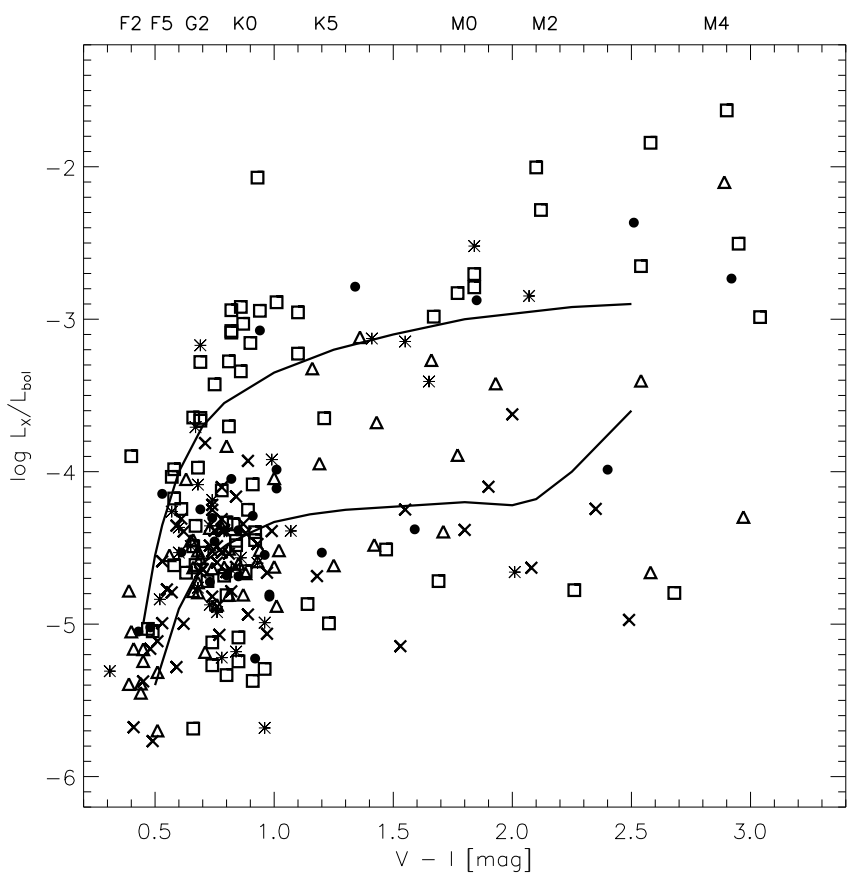

Fig. 5. $\log L_{\mathrm{X}} / L_{\text {bol }}$ vs. $V-I$ of the members of the Local Association (squares), Castor Moving Groups (filled circles), Ursa Major Moving Groups (triangles), Hyades Supercluster (x symbols), and IC 2391 Supercluster (asterisks). The upper continuous line is the median of the Pleiades members. The continuous line at the bottom is the median of the stars of the Hyades.

Montes et al. (2001a). We compiled photometric, spectroscopic, and X-ray data from the literature. We cross-correlated the sample with the Tycho-2, Hipparcos, 2MASS, and RASS databases. Spectroscopic data were taken from López-Santiago (2005) and López-Santiago et al. (2009).

The general properties of the stars for which we analyzed data indicate that there are probably two different age populations. We used the equivalent width of the lithium line $\lambda 6708.8 \AA$ and the X-ray luminosities to constrain the ages of the candidates of the Local Association, which is the moving group containing the youngest stars. The analysis indicates that the contribution of old field stars to the sample of candidates of this moving group is $\sim 50 \%$ if we used the whole sample. A more accurate study using only those stars at $d \leq 40 \mathrm{pc}$, in which our sample of X-ray sources is nearly complete, showed that the contribution of nonmembers is, in fact, approximately $30 \%$. This number must be considered to represent the contamination by old main sequence stars of the Montes et al. (2001a) sample of candidate members of the young moving group. A higher percentage of interlopers among possible members of the Pleiades Moving Group was determined in Famaey et al. (2008). The age of the other moving groups does not permit members to be separated univocally from non-members, since the properties of the candidates are similar to those of field stars. However, we found that the X-ray data can be used to distinguish, at least, between stars of age <200 Myr and older stars.

Our result is consistent with that found for the Hyades Supercluster by Famaey et al. (2007). In that study, the authors concluded that the moving group consist of stars coeval with the Hyades cluster, which presumably formed in the same star formation episode, and old stars with similar space motion. The samples of candidate members of the classical young moving groups $(<650 \mathrm{Myr})$ contain a non-negligible amount of old $(>1 \mathrm{Gyr})$ field stars. With regard to this matter, several studies suggest that the three arm-like shape observed in the $U V$-plane is caused by a non-axisymmetric potential (Famaey et al. 2005) and that the same non-axisymmetric motion affecting old-field stars should also perturb the molecular clouds where star formation occurs (Xu et al. 2006). This possibly explains for the location of the young stellar associations such as TW Hya or $\eta C h a$ inside the Local Association in the $U V$-plane. In addition, this scenario could explain the problem of the apparent lack of post-T Tauri stars close to star-forming regions (Herbig 1978; Soderblom et al. 1998), which is inferred from the increasing number of them identified in loose associations and moving groups (Bubar et al. 2007; Torres et al. 2008; Montes et al. 2009).

We have extended our conclusions for the Local Association to the other young moving groups. The contamination by old stars in the moving groups should be considered in studies of the history of stellar birthrate in the solar neighborhood. Only by using data of different wavelengths, in particular X-ray and optical spectroscopic data, is it possible to distinguish between the two age populations. More detailed studies in this area will enable us to determine univocally the contribution of young stars to the stellar population in the solar vicinity, to compare with the predictions of Galactic models.

Acknowledgements. J. López-Santiago acknowledges financial support by the Marie Curie Fellowship contract No. MTKD-CT-2004-002769 and financial contribution by MERG-CT-2007-046535. The Madrid group acknowledges financial contribution by the Universidad Complutense de Madrid and the Programa Nacional de Astronomía y Astrofísica of the Spanish Ministerio de Educación y Ciencia (MEC), under grants AYA2005-02750 and AYA2008000695 and to the PRICIT project S-0505/ESP-0237 (ASTROCAM) of the Comunidad de Madrid. G. Micela acknowledges financial contribution from contract ASI-INAF I/088/06/0. We thanks the referee for useful comments that allowed to improve our manuscript. This publication makes use of data products from the Two Micron All Sky Survey, which is a joint project of the University of Massachusetts and the Infrared Processing and Analysis Center/California Institute of Technology, funded by the National Aeronautics and Space Administration and the National Science Foundation. This research made use of the SIMBAD database, operated at the CDS, Strasbourg, France.

\section{References}

Affer, L., Micela, G., \& Morel, T. 2008, A\&A, 490, 601

Antoja, T., Figueras, F., Fernndez, D., \& Torra, J. 2008, A\&A, 490, 135

Asiain, R., Figueras, F., Torra, J., \& Chen, B. 1999, A\&A, 341, 427

Barrado y Navascués, D. 1998, A\&A, 339, 831

Bertout, C., \& Genova, F. 2006, A\&A, 460, 499

Bessell, M. S., \& Brett, M. 1988, PASP, 100, 113

Bubar, E. J., King, J. R., Soderblom, D. R., Deliyannis, C. P., \& Boesgaard, A. M. 2007, AJ, 134, 2328

Daemgen, S., Siegler, N., Reid, I. N., \& Close, L. M. 2007, ApJ, 654, 558

Eggen, O. J. 1991, AJ, 102, 2028

Eiroa, C., Garzón, F., Alberdi, A., et al. 2001, A\&A, 365, 110

ESA 1997, The Hipparcos and Tycho Catalogues, ESA SP-1200

Famaey, B., Jorissen, A., Luri, X., et al. 2005, A\&A, 430, 165

Famaey, B., Pont, F., Luri, X., et al. 2007, A\&A, 461, 957

Famaey, B., Siebert, A., \& Jorissen, A. 2008, A\&A, 483, 453

Favata, F., Barbera, M., Micela, G., \& Sciortino, S. 1993, A\&A, 277, 428

Fernández, D., Figueras, F., \& Torres, J. 2008, A\&A, 480, 735

Francis, C., \& Anderson, E. 2009, in press [arXiv: 0901.3503]

Guillout, P., Sterzik, M. F., Schmitt, J. H. M. M., et al. 1998, A\&A, 334, 540

Herbig, G. H. 1978, in Problems of Physics and Evolution of the Universe, ed.

L. V. Mirzoyan (Yerevan: Publishing House of the Armenian Academy of Sciences), 171

Hg, E., Fabricius, C., Makarov, V. V., et al. 2000, A\&A, 355, L27

Hünsch, M., Schmitt, J. H. M. M., Schroeder, K. P., \& Reimers, D. 1996, A\&A, 310,801

King, J. R., Villarreal, A. R., Soderblom, D. R., Gulliver, A. F., \& Adelman, S. J. 2003, AJ, 125, 1980

Klement, R., Fuchs, B., \& Rix, H.-W. 2008, ApJ, 685, 261

López-Santiago, J. 2005, Ph.D. Thesis, Universidad Complutense de Madrid, Spain

López-Santiago, J., Montes, D., Crespo-Chacón, I., \& Fernández-Figueroa, M. J. 2006, ApJ, 643, 1160 
López-Santiago, J., Micela, G., Sciortino, S., et al. 2007, A\&A, 463, 165 López-Santiago, J., Montes D., Gálvez M. C., et al. 2009, A\&A, in preparation Makarov, V. V. 2007, ApJS, 169, 105

Mamajek, E. E., \& Feigelson, E. D. 2001, ASP Conf. Ser. 244, Young Stars Near Earth: Progress and Prospects, ed. R. Jayawardhana, \& Th. Greene, 104

Micela, G., Affer, L., Favata, F., et al. 2007, A\&A, 461, 977

Montes, D., López-Santiago, J., Gálvez, M. C., et al. 2001a, MNRAS, 328, 45 Montes, D., López-Santiago, J., Fernández-Figueroa, M. J., \& Galvez, M. C. 2001b, A\&A, 379, 976

Montes, D., López-Santiago, J., Martínez-Arnáiz, R. M., et al. Proceedings of the The 15th Cool Stars, Stellar Systems and the Sun workshop, held in St Andrews, Scotland, UK, July 21-25, 2008, ed. E. Stempels, Conf. Proc. Ser. Amer. Inst. Phys. (AIP), 1094, 943

Orlov, V. V., Panchenko, I. E., Rastorguev, A. S., \& Yatsevich, A. V. 1995, Astron. Zh., 72, 495
Schmitt, J. H. M. M., Fleming, T. A., \& Giampapa, M. S. 1995, ApJ, 450, 392 Sciortino, S., Favata, F., \& Micela, G. 1995, A\&A, 296, 370

Siess, L., Duffour, E., \& Forestini, M. 2000, A\&A, 358, 593

Skuljan, J., Hearnshaw, J. B., \& Cottrell, P. L. 1999, MNRAS, 308, 731

Soderblom, D. R., \& Mayor, M. 1993, ApJ, 402, 5

Soderblom, D. R., Jones, B. F., Balachandran, S., et al. 1993, AJ, 106, 1059

Soderblom, D. R., King, J. R., Siess, L., et al. 1998, ApJ, 498, 385

Torres, C. A. O., Quast, G. R., da Silva, L., et al. 2006, A\&A, 460, 695

Torres, C. A. O., Quast, G. R., Melo, C. H. F., \& Sterzik, M. F. 2008, ASP Monogr. Public., 5, 757

Xu, Y., Reid, M. J., Zheng, X. W., \& Menten, K. M. 2006, Science, 311, 54

Zhao, J., Zhao, G., \& Chen, Y. 2009, ApJ, in press [arXiv:0902 .1370]

Zickgraf, F.-J., Krautter, J., Reffert, S., et al. 2005, A\&A, 433, 151

Zuckerman, B., \& Song, I. 2004, ARA\&A, 42, 685 